\title{
Association of higher axillary pathologic complete response rate with breast pathologic complete response after neoadjuvant chemotherapy
}

\author{
Jiujun Zhu ${ }^{1 \#}$, Jianbin $\mathrm{Li}^{2 *}$, Zhimin Fan ${ }^{3}$, Haibo Wang ${ }^{4}$, Jianguo Zhang ${ }^{5}$, Yongmei Yin ${ }^{6}$, Peifen Fu ${ }^{7}$, \\ Cuizhi Geng ${ }^{8}$, Feng Jin ${ }^{9}$, Zefei Jiang ${ }^{2}$, Zhenzhen Liu ${ }^{1}$
}

${ }^{1}$ Department of Breast Cancer Center, Affiliated Cancer Hospital of Zhengzhou University; Henan Cancer Hospital, Zhengzhou, China; ${ }^{2}$ Department of Breast Oncology, The Fifth Medical Center of Chinese PLA General Hospital, Beijing, China; ${ }^{3}$ Department of Breast Surgery, The First Hospital of Jilin University, Changchun, China; ${ }^{4}$ Department of Breast Cancer Center, Affiliated Hospital of Medical College Qingdao University, Qingdao, China; ${ }^{5}$ Department of Breast Surgery, the Second Affiliated Hospital of Harbin Medical University, Harbin, China; ${ }^{6}$ Department of Breast Cancer, Jiangsu Province Hospital and Nanjing Medical University First Affiliated Hospital, Nanjing, China; ${ }^{7}$ Department of Breast Center, First Affiliated Hospital, School of Medicine, Zhejiang University, Hangzhou, China; ${ }^{8}$ Department of Breast Cancer Center, Hebei Medical University Fourth Affiliated Hospital and Hebei Provincial Tumor Hospital, Shijiazhuang, China; ${ }^{9}$ Department of Breast Surgery, the First Affiliated Hospital of China Medical University, Shenyang, China

Contributions: (I) Conception and design: Z Liu, Z Jiang; (II) Administrative support: Z Liu, Z Jiang; (III) Provision of study materials or patients: All authors; (IV) Collection and assembly of data: J Zhu, J Li; (V) Data analysis and interpretation: J Zhu; (VI) Manuscript writing: All authors; (VII) Final approval of manuscript: All authors.

\#These authors contributed equally to this work.

Correspondence to: Zhenzhen Liu. Department of Breast Cancer Center, Affiliated Cancer Hospital of Zhengzhou University, Henan Cancer Hospital, No. 127, Dongming Road, Zhengzhou, China. Email: zlyyliuzhenzhen0800@zzu.edu.cn; Zefei Jiang. Department of Breast Oncology, The Fifth Medical Center of Chinese PLA General Hospital, No. 8 East Street, Fengtai District, Beijing, China. Email: jiangzefei@csco.org.cn.

Background: To investigate the association of axillary pathologic complete response (pCR) rate among breast cancer patients with pCR after neoadjuvant chemotherapy (NCT).

Methods: The retrospective clinical data of 1,903 patients who were treated with NCT between March, 2010 and December, 2018, were collected from one Chinese database and analyzed. The correlations between clinicopathological characteristics and breast pCR with axillary pCR were calculated by $\chi^{2}$ test. Binary logistic regression analysis was used for multivariate analysis. The relative risk of positive axillary nodes after NCT in patients with and without breast pCR was analyzed using a Cochran-Mantel-Haenszel $(\mathrm{CMH})$ test stratified by initial $\mathrm{N}$ stage and tumor subtype.

Results: The rate of axillary pCR was increased in the cases with initial cN0, Ki67 high expression, HR+HER2+/HR-HER2+/TN subtypes, and breast pCR. After NCT, the relative risk of nodal disease burden was 4.81 in patients without breast pCR compared with patients with breast pCR. The relative risk of positive nodal status in patients with $\mathrm{cN} 0, \mathrm{cN} 1, \mathrm{cN} 2$, and $\mathrm{cN} 3$ disease without $v$ s. with breast pCR was $6.45,4.88,5.69$ and 6.24 , respectively. The relative risk of positive nodal status in patients with HR+HER2-, HR+HER2+, HR-HER2+, and TN disease was 4.02, 4.50, 3.82 and 4.18, respectively. Of cN0 patients with breast pCR, only 4 out of 44 (9\%) with HER2-positive disease had 1 or 2 axillary lymph node metastases at final surgical pathology compared to 30 out of 98 (31\%) of those without breast pCR. There was no evidence of positive nodal residue among all 21 patients (100\%) with TN disease compared to 65\% (36 of 55) of patients without breast pCR.

Conclusions: Nodal status is strongly correlated with breast pCR after NCT. Patients with initial cN0/1 TN/HER2 positive disease who achieve breast pCR at surgery have a low risk of nodal metastasis. These results suggest that the failure rate of missing positive lymph nodes among those patients was very low and that it is safe for such patients to undergo sentinel lymph node biopsy (SLNB) after NCT. This study also provides a theoretical basis for clinical trials focused on the avoidance of axillary surgery in selected patients. 
Keywords: Breast neoplasms; neoadjuvant chemotherapy (NCT); axillary lymph node; pathologic complete response (pCR)

Submitted Jun 09, 2020. Accepted for publication Jul 29, 2020.

doi: $10.21037 / \mathrm{atm}-20-5172$

View this article at: http://dx.doi.org/10.21037/atm-20-5172

\section{Introduction}

Neoadjuvant chemotherapy (NCT) with or without targeted therapy has been widely recognized and applied in the treatment of locally advanced, triple negative (TN), and human epidermal growth factor receptor 2 (HER2)positive breast cancer. In locally advanced (inoperable) patients, NCT is frequently used to downstage patients from inoperable to operable before surgery or to increase the rate of breast-conserving surgery. Owing to the improvement of molecular typing-guided NCT and the development of targeted therapeutic drugs, the rate of pathologic complete response (pCR) has increased continuously in recent years, especially among patients with TN and HER2-positive breast cancer. Patients who attain pCR have improved event free survival (HR 0.48, 95\% CI: 0.43-0.54) and overall survival (HR 0.36, 95\% CI: 0.31-0.42) than those with residual invasive cancer. The association between pCR and longterm outcomes was strongest in patients with TN breast cancer and in those with HER2-positive, hormone receptor negative tumors who received trastuzumab therapy (1). PCR can not only serve as an alternative indicator of longterm survival, but it can also inform the downstaging treatment strategy for the local control of breast cancer. After NCT, some patients were down staged from positive axillary lymph node (cN1) to clinically negative (cN0). Although with some controversy, it was believed that sentinel node biopsy(SLNB), as opposed to axillary dissection, to be adequate if at least three or more negative sentinel nodes were detected and examined (2).

Recently, the surgical modalities used to manage breast cancer have changed considerably. In the past, for example, mastectomies were mostly performed after NCT; however, some patients now receive breast-conserving surgery instead. However, the treatment strategy for the axilla requires a different approach. For certain patients with initial cN0/1 status, a large number of studies have supported the application of SLNB to avoid unnecessary axillary lymph node dissection (ALND). Owing to its low detection rate (DR) and high false negative rate (FNR), however, the clinical application of SLNB is limited (3). In some breast tumor centers, ALND is performed in most patients, regardless of the response of the lymph nodes to chemotherapy. This unnecessarily deprives many patients who have no residual disease in the axilla of their axillary lymph nodes, and increases the risk of complications such as lymphedema, upper limb numbness, and limited shoulder movement.

Optimizing patient selection is one approach to solving the dilemma posed by SLNB after NCT. The main reason for the controversy about SLNB after NCT is the concern about the missed detection of metastatic lymph nodes. If the patients have no residual tumor in the lymph nodes, there is no risk of missed detection. Therefore, patients with low risk of residual axillary lymph nodes after NCT can be selected for SLNB. Some studies have reported the predictive factors of axillary lymph node status after NCT. Kantor et al. developed a predictive model based on data from the National Cancer Database (NCDB) to identify the individuals who were most likely to obtain axillary pCR after NCT among patients with initial node-positive disease (4). They concluded that age, molecular subtype, histological grade, histological type, and initial node stage were all significant independent predictors of post-NCT axillary pCR in adjusted analysis. However, few reports have evaluated the correlation of pCR in the breast and axillary lymph nodes. Moreover, due to the high rate of pCR after NCT in some subtypes, the necessity for patients with breast pCR to undergo surgery is also questionable. Several studies have attempted to avoid breast surgery in selective patients after NCT (5). But there is no report on the results based on the Asian population database. Therefore, the question arises as to whether SLNB can be avoided for patients in certain subtypes in Asian patients.

The present study aimed to identify patients who are suitable for SLNB after NCT safely by comparing the difference in risk of axillary node metastasis in patients with and without breast pCR in Asian patients. Furthermore, it also provides a theoretical basis for future research on avoiding axillary surgery after NCT. We present the 


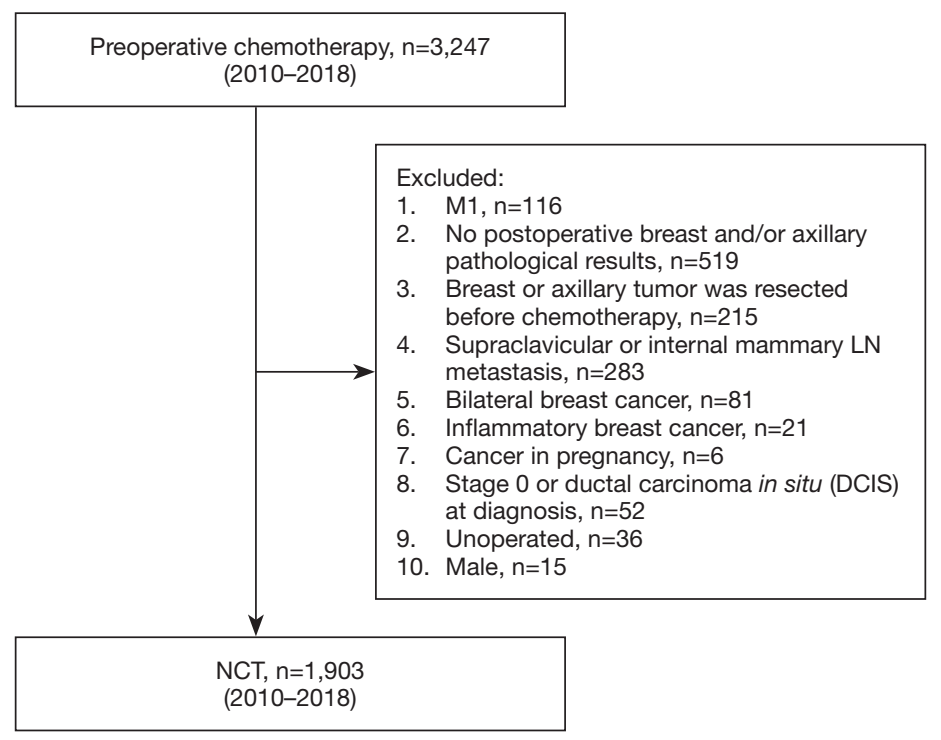

Figure 1 Patient flow diagram.

following article in accordance with the STROBE reporting checklist (available at http://dx.doi.org/10.21037/atm-205172).

\section{Methods}

The data in this study were obtained from the Chinese Society of Clinical Oncology (CSCO) Breast Cancer Database Collaboration Group. The CSCO database contains anonymized breast cancer cases from nine hospitals representing all regions of China. We identified all patients with primary breast cancer who had received preoperative chemotherapy between March, 2010, and December, 2018. The collected data included sex, age at diagnosis, menstrual status, location of primary breast tumor, initial clinical (cTNM) stage, estrogen receptor (ER) status, progesterone receptor (PR) status, HER2 status, Ki-67 status, surgical mode, and postoperative breast and axillary pathology.

The inclusion criteria were as follows: (I) invasive breast cancer confirmed by core needle biopsy before chemotherapy. (II) cTNM stage according to the 7 th edition of American Joint Cancer Commission (AJCC) cTNM staging before treatment with NCT available. Initial $\mathrm{T}$ stage was determined by physical examination. The axillary nodal status was determined by physical examination and ultrasound. If no suspicious enlarged lymph nodes were found during physical examination, the patient was defined as clinically node negative (cN0). If suspicious lymph nodes detected by ultrasound were confirmed without tumor metastasis by additional core needle biopsy or fineneedle aspiration cytology, the patient was also defined as cN0. (III) ER, PR, HER2, and Ki67 were detected before chemotherapy. (IV) The patient received chemotherapy before the operation. (V) After the completion of NCT, the patient underwent breast and axillary surgery according to the local treatment standards. (VI) Postoperative pathology of breast and axillary lymph nodes available. Patients meeting any of the following criteria were excluded from the study: (I) male; (II) bilateral breast cancer; (III) distant metastasis (M1); (IV) supraclavicular or internal mammary lymph node metastasis; (V) inflammatory breast cancer; (VI) cancer in pregnancy; (VII) stage 0 or ductal carcinoma in situ (DCIS) at diagnosis; (VIII) primary breast or axillary tumor resected before chemotherapy; (IX) simultaneous loss of ER, PR, and HER2; (X) absence of postoperative breast and lymph node pathology; or (XI) no operation. A total of 3,247 patients were collected. After screening, 1,903 patients were deemed to be eligible and included in the final analysis (Figure 1).

The study was conducted in accordance with the Declaration of Helsinki (as revised in 2013). Data were obtained after this study had been approved by the Ethical Review Committee of the Affiliated Cancer Hospital of Zhengzhou University (No. 2019001). As this study was retrospectively designed, informed consent was waived by the Affiliated Cancer Hospital of Zhengzhou University. 
The status of ER, PR, HER2, and Ki67 was evaluated by immunohistochemistry (IHC) (6). ER/PR positive (+): $\geq 1 \%$ of tumor cells with nuclear staining. $\mathrm{ER}+$ or $\mathrm{PR}+$ were collapsed into a single hormone receptor positive (HR+). HER2 positive was determined according to the American Society of Clinical Oncology (ASCO) guidelines (7). An IHC score of $3+$ was considered positive, as was a $2+$ IHC score with the results of fluorescence in situ hybridization (FISH) overruled. Ki67 expression was classified as low $(<30 \%)$ or high $(>30 \%)$ according to the ratio of nuclear positive cells to all tumor cells in 10 high-power visual fields. The representative IHC and FISH images of ER, PR, HER2 and Ki67 were shown in the Figure S1. The patients were divided into four subtypes according to HR and HER2 status: HR+HER2-, HR+HER2+, HR-HER2+, and HR-HER2- (TN). PCR was defined as the absence of tumor cells in the breast (breast pCR) or the axillary lymph nodes (axillary pCR) after NCT (1).

\section{Statistical analyses}

Statistical analyses were performed with GraphPad Software 5.0 and Statistical Package for the Social Sciences software (SPSS, version 23, IBM, Armonk, NY, USA). Categorical variables were compared using the $\chi^{2}$ test. Binary logistic regression analysis was used for multivariate analysis. Relative risk (RR) for positive axillary nodes after NCT in patients with and without breast $\mathrm{pCR}$ were analyzed with a Cochran-Mantel-Haenszel (CMH) test stratified by initial $\mathrm{N}$ stage and tumor subtype. A two-sided $\mathrm{P}$ value of $<0.05$ was considered to be statistically significant.

\section{Results}

\section{Patient characteristics}

A total of 1,903 female breast cancer patients (age: median, 46 , range, 20-77 years) who received NCT and subsequent surgical resection between March, 2010, and December, 2018. Two-thirds (65\%) of the patients were premenopausal at diagnosis. At presentation, 1,252 patients (67\%) had cT1/2 disease and 1,151 (61\%) had cN0/1 disease. The distribution across the approximated molecular subtypes was 43\% (817) HR+HER2-, 22\% (420) HR+HER2+, 17\% (310) HR-HER2+, and 18\% (335) TN breast cancer. The average number of axillary lymph nodes was 15.4 (average positive, 2.7) at surgery following NCT. Axillary pCR and breast pCR were achieved by $45 \%$ (852) and 25\% (469) of patients, respectively. Of the patients who achieved breast pCR, $340(72 \%)$ also achieved axillary pCR (Table 1, Figure 2A). A higher rate of axillary pCR was observed among patients with HER2+ $(56 \%)$ and TN (56\%) disease than among those with HR+HER2- (30\%) disease. The HER2+ group was stratified according to HR- and HR+ status, and a slightly higher rate of axillary pCR was seen in the HR-HER2 + subgroup compared with the HR+HER2+ subgroup (61\% vs. $52 \%)$.

\section{Correlations between axillary pCR and clinicopathological characteristics}

Univariable analysis showed that the clinicopathological features associated with a higher probability of achieving axillary pCR were initial cN0 stage, Ki67 high expression, HER2+/TN subtypes, and breast pCR. Multivariable analysis after adjusting for confounders revealed that the probability of showing axillary pCR was still increased in patients with cN0 stage, Ki67 high expression (OR 1.58, 95\% CI: 1.20-2.08, $\mathrm{P}<0.01), \mathrm{HR}+\mathrm{HER} 2+(\mathrm{OR} 2.27,95 \%$ CI: 1.70-3.03, $\mathrm{P}<0.01) / \mathrm{HR}-\mathrm{HER} 2+(\mathrm{OR} 3.09$, 95\% CI: 2.21-4.33, $\mathrm{P}<0.01) / \mathrm{TN}(\mathrm{OR} 2.12,95 \%$ CI: $1.50-3.00$, $\mathrm{P}<0.01)$ subtypes, and breast pCR (OR 4.38, 95\% CI: 3.34 5.73, $\mathrm{P}<0.01)$ (Table 1, Figure 2B).

\section{Association between axillary $p C R$ and breast pCR}

The rate of axillary pCR was significantly higher in patients with breast pCR than in those without $73 \%$ vs. $36 \%, \mathrm{P}<0.05)$. Table 2 shows the relative risk of residual axillary nodal metastases, as stratified by clinical $\mathrm{N}$ stage and biological subtype. Relative risk ratio analyses showed that after NCT, the relative risk of nodal disease burden in patients without breast pCR was 4.81 (95\% CI: 3.82 6.06, $\mathrm{P}<0.01)$ compared with patients with breast $\mathrm{pCR}$. At presentation, patients without breast $\mathrm{pCR}$ across all groups had a higher risk of having positive axillary nodes after NCT. The relative risk of positive nodal status in patients with $\mathrm{cN} 0, \mathrm{cN} 1, \mathrm{cN} 2$, and $\mathrm{cN} 3$ disease without versus with breast pCR was 6.45 (95\% CI: 3.12-13.32, $\mathrm{P}<0.01$ ), 4.88 (95\% CI: 3.38-7.04, $\mathrm{P}<0.01$ ), 5.69 (95\% CI: 3.74-8.66, $\mathrm{P}<0.01$ ), and 6.24 (95\% CI 3.23-12.03, $\mathrm{P}<0.01$ ), respectively. A significant increase in relative risk was also observed between patients within each tumor subtype who presented without $v s$. with breast $\mathrm{pCR}$. The relative risk in patients with HR+HER2-, HR+HER2+, HR-HER2+, and TN disease was 4.02 (95\% CI: 2.70-6.00, $\mathrm{P}<0.01$ ), 4.50 
Table 1 Baseline patient characteristics and corresponding axillary pCR rates (\%)

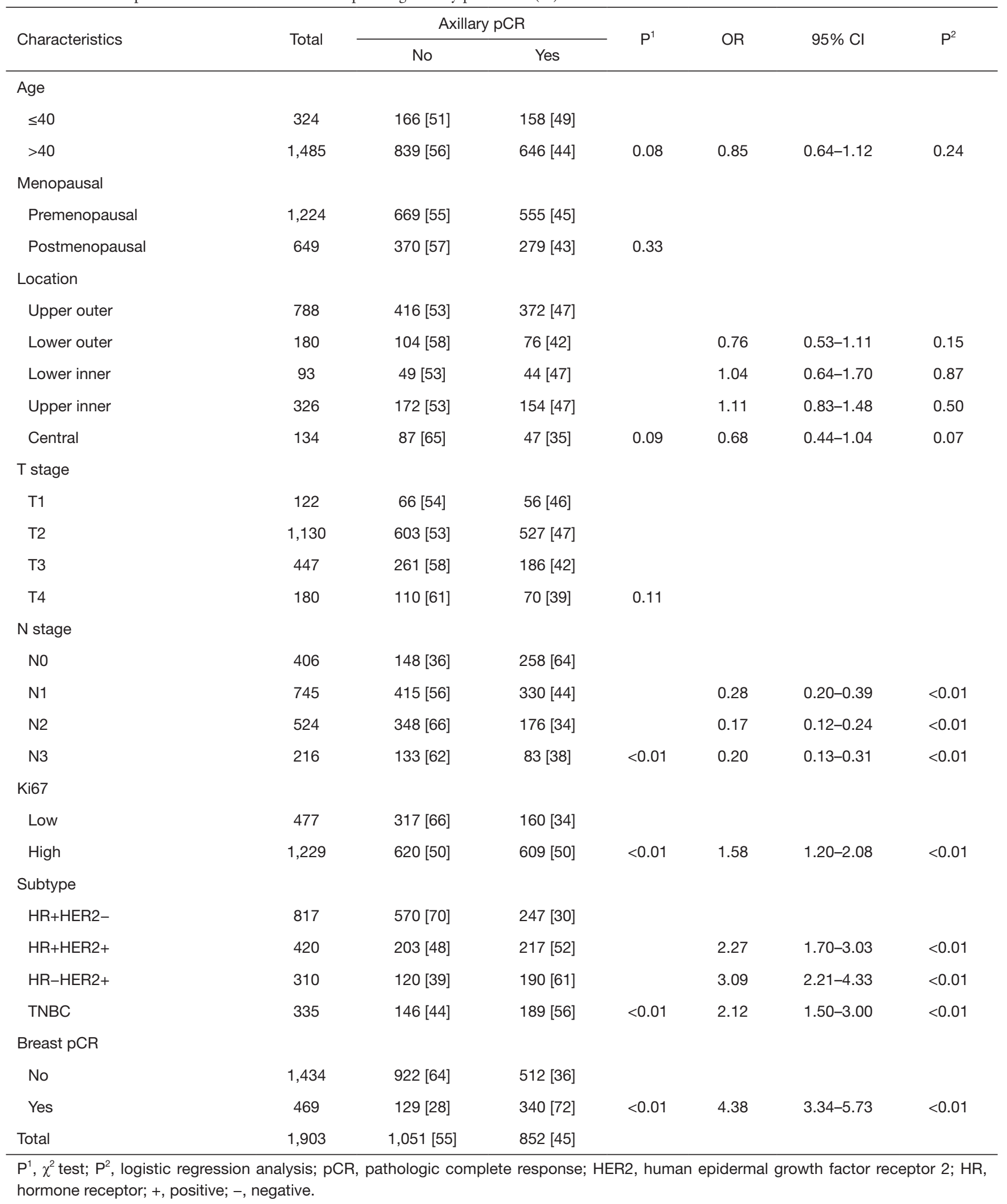



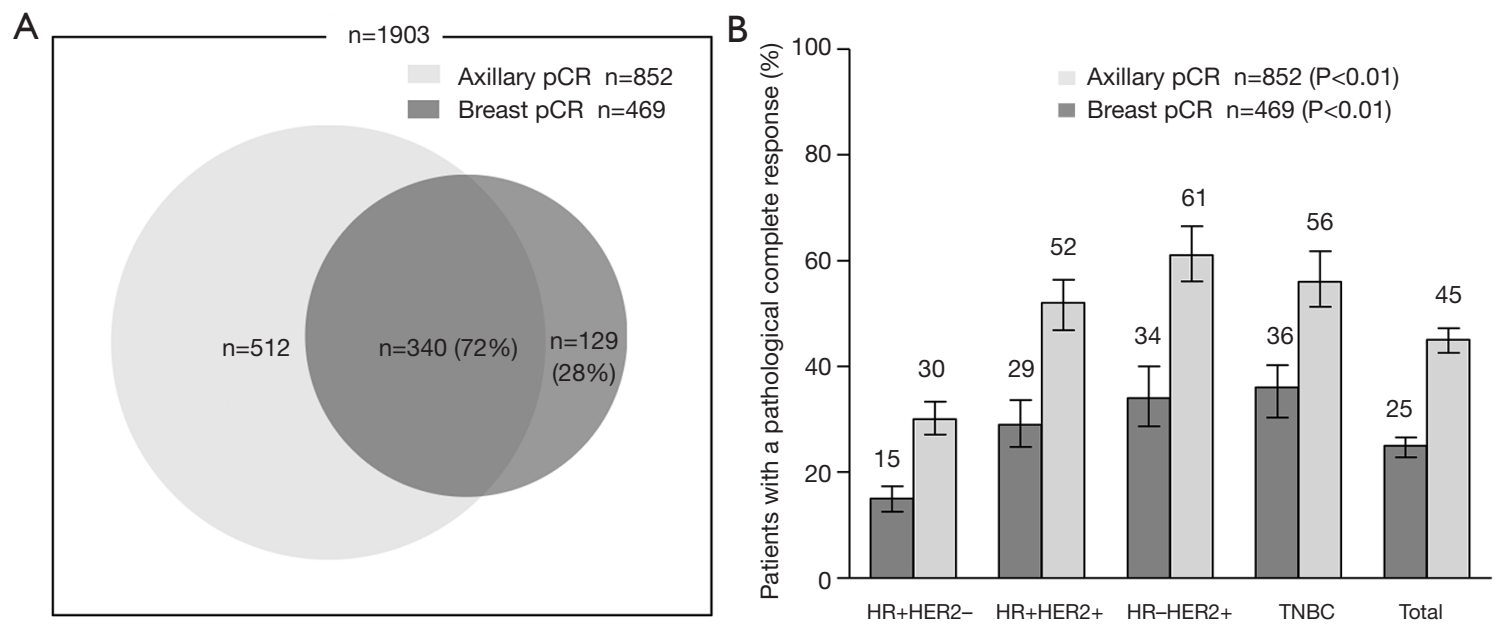

Figure 2 PCR in axillary and breast. (A) Association of the incidence rate of axillary pCR in patients who achieved breast pCR. (B) Breast pCR and axillary pCR outcomes after NCT among patients with different breast cancer subtypes (\%). pCR, pathologic complete response; NCT, neoadjuvant chemotherapy.

(95\% CI: 2.79-7.25, $\mathrm{P}<0.01$ ), 3.82 (95\% CI: 2.20-6.62, $\mathrm{P}<0.01)$, and 4.18 (95\% CI: 2.52-6.93, $\mathrm{P}<0.01)$ respectively (Table 2).

Because of the low rate of axillary pCR after NCT in patients with $\mathrm{cN} 2 / 3$ (34\% and $38 \%$ ) disease, only the patients with initial $\mathrm{cN} 0$ and $\mathrm{cN} 1$ status within each tumor subtype were included in subsequent evaluation. Of the patients who had $\mathrm{cN} 0$ disease at presentation, $64 \%$ had an axillary pCR overall. Of those, the nodal negative rate was higher in patients with breast pCR than without $(89 \% v s$. $57 \%, \mathrm{P}<0.01)$. This trend was also demonstrated in each molecular subtype. The nodal negative rate in patients with HR+HER2-, HR+HER2+, HR-HER2+ and TN disease was $75 \%$ vs. $46 \%(\mathrm{P}=0.01), 91 \%$ vs. $66 \%(\mathrm{P}<0.01), 91 \%$ vs. $73 \%(\mathrm{P}<0.01)$, and $100 \%$ vs. $65 \%(\mathrm{P}<0.01)$, respectively. In patients with $\mathrm{cN} 1$ disease, $44 \%$ had axillary pCR overall. Of these patients, the nodal negative rate was also significantly higher for HR+HER2- (68\%), HR+HER2+ (75\%), HRHER2+ (74\%), and TN (76\%) patients with breast pCR compared with those with residual breast disease (26\%, $38 \%, 53 \%$, and $44 \%$, respectively) (Table 3).

Table 4 shows further analysis of the number of residual positive lymph nodes at final pathology. In the case of $\mathrm{cN} 0$ patients with breast pCR, only 4 out of 44 (9\%) with HER2+ disease had 1 or 2 axillary lymph node metastases at final surgical pathology compared with 30 of 98 (31\%) patients without breast pCR. Among the 4 patients, 3 had 1 positive lymph node and 1 had 2 positive lymph nodes. No evidence of positive nodal residue was found in the 21 patients
(100\%) with TN disease, compared to $65 \%$ (36 of 55 ) patients without breast pCR at surgery. Of the patients with cN0 disease who achieved breast pCR, patients with $\geq 3$ positive lymph nodes were found only in the HR+HER2group ( $15 \%, 3$ of 20 ; overall $4 \%$ ), compared to rates ranging from $9 \%$ to $30 \%$ across all subtypes of patients without breast pCR (overall $23 \%$ ). Ninety patients (43\%) with cN1 and HER2-positive disease were found to have obtained breast pCR. Of them, 23 did not achieve axillary pCR (23 of 90, 26\%). Among these 23 patients, 12 had 1, 5 had 2, and $6 \mathrm{had} \geq 3$ positive lymph nodes. Overall, we found no significant difference in the rate of axillary pCR between the HER2-positive (67 of 90, 74\%) and TN (31 of 41, 76\%) groups who achieved breast pCR. For patients with $\mathrm{cN} 1$ disease, the rate of residual disease in $\geq 3$ nodes ranged from $6 \%$ to $15 \%$ (overall $10 \%$ ) and $25 \%$ to $40 \%$ (overall $34 \%$ ) in patients with and without breast pCR, respectively (Table 4).

\section{Discussion}

Over the past decade, improvements in the efficacy of chemotherapy and HER2-targeted therapies have led to an increase in the pCR rate of breast cancer. The response rate has been found to be slightly higher in the lymph nodes than in the breast, with a reported axillary pCR rate of 22-44\% (8). Typically manifested in TN and HER2positive disease, the rate of axillary pCR has reached as high as $40 \%$ to $74 \%$ (9). Patients who obtain axillary pCR also have reported a significantly higher survival rate than 
Table 2 Relative risk of positive axillary nodes after NCT in patients with and without breast pCR (\%)

\begin{tabular}{|c|c|c|c|c|c|c|c|}
\hline Characteristics & Breast pCR & Total & \multicolumn{2}{|c|}{ Axillary pCR } & $\mathrm{RR}$ & $95 \% \mathrm{Cl}$ & $\mathrm{P}$ \\
\hline \multirow[t]{2}{*}{ No } & No & 321 & 139 [43] & $182[57]$ & & & \\
\hline & Yes & 85 & $9[11]$ & 76 [89] & 6.45 & $3.12-13.32$ & $<0.01$ \\
\hline $\mathrm{N} 1$ & No & 560 & 364 [65] & 196 [35] & & & \\
\hline \multirow[t]{2}{*}{ N2 } & No & 386 & 297 [77] & 89 [23] & & & \\
\hline & Yes & 138 & $51[37]$ & 87 [63] & 5.69 & $3.74-8.66$ & $<0.01$ \\
\hline \multirow[t]{2}{*}{ N3 } & No & 157 & 115 [73] & $42[27]$ & & & \\
\hline & Yes & 59 & 18 [31] & 41 [69] & 6.24 & $3.23-12.03$ & $<0.01$ \\
\hline \multirow[t]{2}{*}{ HR+HER2- } & No & 696 & 519 [75] & 177 [25] & & & \\
\hline & Yes & 121 & 51 [42] & $70[58]$ & 4.02 & $2.70-6.00$ & $<0.01$ \\
\hline \multirow[t]{2}{*}{ HR+HER2+ } & No & 298 & 174 [58] & 124 [42] & & & \\
\hline & Yes & 122 & 29 [24] & $93[76]$ & 4.50 & $2.79-7.25$ & $<0.01$ \\
\hline \multirow[t]{2}{*}{ HR-HER2+ } & No & 204 & 99 [49] & $105[51]$ & & & \\
\hline & Yes & 106 & 21 [20] & $85[80]$ & 3.82 & $2.20-6.62$ & $<0.01$ \\
\hline \multirow[t]{2}{*}{ TNBC } & No & 216 & 119 [55] & 97 [45] & & & \\
\hline & Yes & 119 & 27 [23] & $92[77]$ & 4.18 & $2.52-6.93$ & $<0.01$ \\
\hline
\end{tabular}

Adjusted $\mathrm{RR}_{\mathrm{N}}=5.49,95 \% \mathrm{Cl}$ : 4.31-6.99, $\mathrm{P}<0.01$; adjusted $\mathrm{RR}_{\text {Subtype }}=4.13,95 \% \mathrm{Cl}: 3.26-5.24, \mathrm{P}<0.01$; pCR, pathologic complete response; RR, relative risk; HER2, human epidermal growth factor receptor 2; HR, hormone receptor; +, positive; -, negative.

Table 3 Axillary pCR outcome after NCT for $\mathrm{cN} 0$ and $\mathrm{cN} 1$ between the different breast cancer subtypes (\%)

\begin{tabular}{|c|c|c|c|c|c|c|c|c|c|c|}
\hline \multirow{3}{*}{ Subtype } & \multicolumn{5}{|c|}{ No } & \multicolumn{5}{|c|}{$\mathrm{N} 1$} \\
\hline & \multicolumn{2}{|c|}{ Breast pCR } & \multirow{2}{*}{$P$} & \multicolumn{2}{|l|}{ Overall } & \multicolumn{2}{|c|}{ Breast pCR } & \multirow{2}{*}{$P$} & \multicolumn{2}{|c|}{ Overall } \\
\hline & No & Yes & & $\mathrm{pCR}$ & $\mathrm{P}$ & No & Yes & & $\mathrm{pCR}$ & $P$ \\
\hline HR+HER2- & 75/163 [46] & $15 / 20[75]$ & 0.01 & 90/183 [49] & & $71 / 270$ [26] & $36 / 53$ [68] & $<0.01$ & 107/323 [33] & \\
\hline HR+HER2+ & $33 / 50[66]$ & 20/22 [91] & 0.03 & $53 / 72$ [74] & $<0.01$ & 51/136 [38] & $36 / 48$ [75] & $<0.01$ & 87/184 [47] & 0.36 \\
\hline TNBC & $36 / 55$ [65] & $21 / 21$ [100] & $<0.01$ & $57 / 76$ [75] & $<0.01$ & $32 / 73[44]$ & $31 / 41[76]$ & $<0.01$ & 63/114 [55] & 0.01 \\
\hline Total & $179 / 316$ [57] & 76/85 [89] & $<0.01$ & 255/401 [64] & & 192/551 [35] & 134/184 [73] & $<0.01$ & $326 / 735[44]$ & \\
\hline
\end{tabular}

pCR, pathologic complete response; HER2, human epidermal growth factor receptor 2; HR, hormone receptor; +, positive; -, negative. 
Table 4 Number of pathologic nodal residual tumors according to breast pCR and subtype (\%)

\begin{tabular}{|c|c|c|c|c|c|c|c|c|c|c|}
\hline Characteristics & \multicolumn{4}{|c|}{ Breast pCR } & Total & \multicolumn{4}{|c|}{ No breast pCR } & Total \\
\hline \multicolumn{11}{|l|}{ No } \\
\hline HR+HER2- & 15 [75] & $2[10]$ & $0[0]$ & 3 [15] & 20 & 74 [45] & 23 [14] & $17[10]$ & 49 [30] & 163 \\
\hline HR+HER2+ & 20 [91] & 2 [9] & $0[0]$ & $0[0]$ & 22 & 33 [66] & $5[10]$ & $1[2]$ & 11 [22] & 50 \\
\hline TNBC & 21 [100] & $0[0]$ & $0[0]$ & $0[0]$ & 21 & 36 [65] & $6[11]$ & 8 [15] & 5 [9] & 55 \\
\hline Total & 76 [89] & $5[6]$ & $1[1]$ & $3[4]$ & 85 & $178[56]$ & 38 [12] & 28 [9] & 72 [23] & 316 \\
\hline \multicolumn{11}{|l|}{ N1 } \\
\hline $\mathrm{HR}+\mathrm{HER} 2-$ & 36 [68] & $3[6]$ & $6[11]$ & 8 [15] & 53 & 71 [26] & 60 [22] & 32 [12] & 107 [40] & 270 \\
\hline TNBC & $31[76]$ & $4[10]$ & 1 [2] & 5 [12] & 41 & $32[44]$ & 16 [22] & $6[8]$ & 19 [26] & 73 \\
\hline Total & 134 [73] & 19 [10] & $12[7]$ & 19 [10] & 184 & 193 [35] & 114 [21] & 57 [10] & 187 [34] & 551 \\
\hline
\end{tabular}

pCR, pathologic complete response; HER2, human epidermal growth factor receptor 2; HR, hormone receptor; +, positive; -, negative.

those with residual nodal disease (9). Consistent with previous studies, this population-based study included patients in China who received NCT and showed that the rate of axillary pCR was $45 \%$. Breast pCR was achieved by $25 \%$ of patients, $72 \%$ of whom obtained axillary pCR. A higher rate of axillary pCR were seen in aggressive TN and HER2-positive tumors compared with HR+HER2disease. Many recent studies have reported the predictive factors of axillary pCR after NCT. For example, a singleinstitution study from the University of Texas MD Anderson Cancer Center (MDACC) showed that patients who achieved axillary pCR were more likely to have TN and HER2-positive disease, high-grade tumor, lower clinical and pathologic T stage, and pCR in the breast (9). We confirmed that lower clinical N stage, TN and HER2positive disease, high expression of Ki67, and breast pCR were all significant independent predictors of axillary pCR in adjusted analysis. Our findings were also consistent with those of a study from South Korea (10).

Further analysis of the correlation between pCR in the breast and axilla showed that breast pCR obtained after NCT was strongly correlated with axillary pCR, especially in the HER2-positive and TN subtypes. The rate of axillary pCR in patients with no residual tumor in the breast was significantly higher than that in patients with residual tumors $(73 \%$ vs. $36 \% \mathrm{P}<0.05)$. The relative risk of axillary lymph node metastasis after NCT in patients with residual breast tumors was 4.81 (95\% CI: 3.82-6.06, $\mathrm{P}<0.01)$ times higher than that in patients without.

The local treatment of initial $\mathrm{cN} 2$ and $\mathrm{cN} 3$ patients after NCT should be approached cautiously and conservatively. In our report, the nodal disease burden was high, with axillary pCR achieved only in $34 \%$ and $38 \%$ of these patients. Also, because the NCCN and other guidelines suggest that those patients should receive ALND and following radiotherapy (11), we will mainly discuss N0 and N1 patients below.

The overall rate of axillary pCR in the cN0 and cN1 patients in our study was $64 \%$ and $44 \%$, respectively. Compared with the breast pCR group, the relative risk of axillary metastasis in the non-breast pCR group was 6.45 (43\% vs. $11 \%, 95 \%$ CI: $3.12-13.32, \mathrm{P}<0.01$ ) and 4.88 times higher $(65 \%$ vs. $27 \%$ CI: $3.38-7.04, \mathrm{P}<0.01)$ (Table 2). These results are consistent with those of a prospective single-institution study from MDACC, which included 527 consecutive patients with HER2-positive and TN (T1/ $\mathrm{T} 2$ and N0/N1) cancer, and aimed to evaluate the risk of axillary metastases in patients who achieve breast pCR after NCT (12). They found that patients without breast pCR had a relative risk of positive lymph nodes of 7.4 compared with those with breast pCR. Among 116 of 290 (40.4\%) patients with TN or HER2-positive disease and initial 
cN0 status and who obtained breast pCR, $100 \%$ had no lymph node metastases at surgery. In 237 cases with cN1, 77 had breast pCR, of whom 69 achieved axillary pCR (89.6\%). The relative risk of axillary metastasis in patients without $v s$. with breast pCR was 14.0 in $\mathrm{cN} 0$ and 5.3 in $\mathrm{cN} 1$ disease. Inconsistent with the MDACC report, we also included patients with initial cT3/T4 stage and patients with HR+HER2- disease. This may possibly account for the lower rate of axillary pCR and the high relative risk of axillary metastasis in patients without breast pCR in our study compared with those in the MDACC study.

Combined analysis with molecular subtypes found that the proportion of axillary pCR in the breast pCR subgroup was significantly higher than in that without breast pCR across all subtypes. At presentation, if patients with TN and HER2-positive disease had breast pCR after NCT, the likelihood of no residual nodal disease was greater than $90 \%$ in those with initial $\mathrm{cN} 0$ status and around $75 \%$ in those with initial $\mathrm{cN} 1$ status. Consistent with the reports from the NCDB and UK databases $(13,14)$, a relatively low rate of nodal disease was demonstrated in patients with initial cN0/1 and TN or HER2-positive disease who achieved breast pCR after NCT. Additionally, patients with T4 status were also included in our study, which was more consistent with the characteristics of real-world data.

Here, we will summarize two important clinical potential implications of our study. Our report shows that SLNB after NCT is safe for selected patients. Although SLNB has become the standard staging technique for initial $\mathrm{cN} 0$ patients, there is currently no consensus on the optimal time (before or after chemotherapy) of SLNB for patients who receive NCT. The NCCN Breast Cancer Guidelines V1 and V2 (2017) recommended that, for patients with initial cN0 disease who undergo NCT, it was feasible to perform SLNB before or after chemotherapy; however, the V3 guidelines changed to recommend SLNB after chemotherapy (11). In the St. Gallen International Expert Consensus of Early Breast Cancer 2017, despite the panel strongly believing SLNB to be appropriate (95.7\%) and favoring that biopsy be carried out after chemotherapy (60\%), $20 \%$ of experts voted for SLNB before NCT, and $16.7 \%$ believed that it could be carried out either before or after neoadjuvant treatment (2). The main reason for the differences in opinion was the concern about positive lymph nodes being missed. After deliberate consideration, we found that the failure rate of positive lymph nodes for the entire cohort was positively correlated with individual risk of axillary involvement and false negative rate (FNR) after NCT. Our study suggests that the proportions of initial cN0 patients with TN and HER2-positive disease with residual positive lymph nodes were $0 \%$ and $9 \%$, respectively. Therefore, the probability of missing positive lymph nodes in those patients was much lower than $10 \%$, which is a widely accepted cut-off value.

There is more controversy surrounding the use of SLNB in patients who present with initial clinical positive lymph nodes and have a clinical response with downstaging to clinical negative lymph nodes. In previous retrospective studies, the rate of FNR ranged from $5 \%$ to $25 \%(15-17)$. The overall rate of FNR in the prospective studies SENTINA (18), ACOSOG Z1071 (19), and SN FNAC (20) was $14.2 \%, 12.6 \%$, and $8.4 \%$, respectively. The high FNR of SLNB after NCT can still be seen. The FNR can be reduced by improving the SLNB technique and using targeted axillary dissection (TAD), which includes marking positive lymph nodes with titanium clips or radioactive seeds before NCT and then locating and removing the marked nodes at surgery, using double tracers, and removing three or more sentinel lymph nodes $(18,19,21)$. Further, we can optimize the selection of patients who are more suitable for SLNB after chemotherapy. Ideally, surgeons should be able to predict which patients will respond better to chemotherapy and are more likely to obtain axillary pCR after NCT, thus confidently minimizing the risk of missing positive lymph nodes. Our study suggests that the proportion of axillary residual metastatic lymph nodes was about $25 \%$ in patients who obtained breast pCR who had initial cN1 TN/HER2-positive disease before NCT. Of those patients, even assuming a high FNR of $30 \%$, the failure rate of missing positive lymph nodes for the entire cohort would have been $7.5 \%$, which is an acceptable value. We can conclude that only a small number of patients who had breast pCR with initial cN1 TN/HER2-positive disease could have ypN-positive disease, so it is safe to perform SLNB for those patients post NCT.

This study provides good theoretical support for future clinical trials focused on certain subgroups of patients for whom axillary surgery could be completely omitted after NCT. Although SLNB is a minimally invasive surgery staging technique for axillary lymph nodes compared with ALND, surgical trauma and complications still exist to a certain degree. At present, some clinical trials, such as the ongoing INSEMA study (NCT02466737) and the SOUND study (NCT02167490), are exploring the possibility of not using axillary surgery in the treatment of particular subsets of patients. These studies were designed 
to determine whether the use of sentinel lymph node surgery could be avoided in patients with negative axillary nodes with ultrasound examination (22). They represent a new attempt for initial $\mathrm{cN} 0$ patients to further realize axillary downstaging surgery on the basis of SLNB instead of ALND. According to a retrospective analysis of the Netherlands Cancer Registry database, the rate of axillary pCR in patients with initial $\mathrm{cN} 0$ and breast pCR was 97.7\%. Axillary pCR was strongly correlated with breast cancer subtype in those patients, with an axillary pCR rate of $93.3 \%, 98.4 \%, 100 \%$, and $98.5 \%$ in HR+HER2-, HR+HER2+, HR-HER2+, and TN disease, respectively (14). In this study, we observed that there were fewer initial cN0 patients with nodal metastasis after NCT, with an axillary pCR rate of $89 \%$, especially in patients with TN $(100 \%)$ and HER2-positive (91\%) disease. The findings of our study combined with those from previous research make avoiding axillary surgery possible and credible in certain patients after NCT, which will help to reduce postoperative complications, improve patients' quality of life, and reduce medical costs.

As can be seen above, identifying breast pCR before surgery appears to be very important in guiding the procedure for axillary surgery given the previously shown significant correlation between breast and axillary pCR. Early conventional and functional breast imaging techniques, including ultrasound, mammography, MRI, and positron emission tomography (PET), are incapable of accurately distinguishing patients with residual breast disease from those without (23-25). However, imagingguided minimally invasive biopsy techniques might have the potential to overcome this impediment. MDACC reported a prospective single-institution study, which enrolled 40 patients with T1-3N0-3 TN or HER2-positive cancer who received NCT. Responsive patients underwent ultrasound or mammography-guided fine-needle aspiration and vacuum-assisted core biopsy of the initial breast tumor region before surgery. Combined fine-needle aspiration and vacuum-assisted core biopsy demonstrated an accuracy of $98 \%$, and a FNR of $5 \%$ in predicting residual tumors (26). Based on the continuous improvement in the rate of breast pCR and the great potential of imaging-guided minimally invasive biopsy in accurately identifying patients with breast pCR after NCT, the necessity of breast surgery for breast pCR patients has also been questioned. An increasing number of prospective clinical trials have focused on avoiding breast surgery in certain patients post NCT. At present, eliminating breast surgery as part of the treatment strategy is not the standard. However, the results of these trials, once reliable and available, may support the omission of breast surgery from the treatment of certain group of patients with breast pCR. In turn, our results could then be used to promote research on the elimination of axillary surgery from the treatment strategies of specific groups of patients ( $\mathrm{cN} 0 / 1$ TN or HER2-positive disease with breast pCR).

The advantage of this study on the correlation between breast and axillary pCR among an Asian population is that a large number of patients were enrolled from the CSCO breast cancer database, which contains registered cases from 9 hospitals representing every region in China. Nevertheless, this research does have some limitations. Firstly, it is a retrospective study based on a database. The number of excluded patients as a consequence of missing data, such as unknown ER, PR, or HER2 status, postoperative pathological tumor and node stage, and the lack of detail about chemotherapy, could have affected the results. Secondly, some patients did not undergo imagingguided biopsy of lymph nodes which were negative in clinical physical examination but suspicious in imaging examination. This means that some initial $\mathrm{cN} 1$ patients were underestimated and identified as $\mathrm{cN} 0$, thus affecting the results. Thirdly, the pathological results came from the pathological laboratories of many centers, and there was a lack of unified certification in the central laboratories. However, these centers are the hospitals with the highest medical quality in China, and their pathology laboratories have also been uniformly certified by the Pathological Quality Control and Evaluation Center of the National Health Commission of the People's Republic of China (PQCC). Therefore, the bias stemming from the results of different centers is minute and can be ignored. Despite the above limitations, this study is more consistent with and better represents real-world patients than some other studies. Moreover, the large sample size of our study also in part made up for its other shortcomings.

In conclusion, there is a strong correlation between breast pCR and axillary metastasis after NCT. Patients with initial cN0/1 TN/HER2-positive disease have low risk of nodal metastases if they achieve breast pCR at surgery. The failure rate of missing positive lymph nodes for the specific subset of patients (cN0/1 TN/HER2-positive disease with breast $\mathrm{pCR}$ ) was very low and it is safe for those patients to undergo SLNB post NCT. Even in those patients with initial $\mathrm{cN} 0$ disease, the failure rate was much lower and acceptable, which makes avoiding the use of axillary surgery after NCT possible. This study also provides a theoretical 
basis for the clinical trial focus on avoiding axillary surgery safely in selected patients.

\section{Acknowledgments}

We are grateful to Dr. Yizhou Jiang for his help with the preparation of the manuscript.

Funding: None.

\section{Footnote}

Reporting Checklist: The authors have completed the STROBE reporting checklist. Available at http://dx.doi. org/10.21037/atm-20-5172

Data Sharing Statement: Available at http://dx.doi. org/10.21037/atm-20-5172

Conflicts of Interest: All authors have completed the ICMJE uniform disclosure form (available at http://dx.doi. org/10.21037/atm-20-5172). The authors have no conflicts of interest to declare.

Ethical Statement: The authors are accountable for all aspects of the work in ensuring that questions related to the accuracy or integrity of any part of the work are appropriately investigated and resolved. The study was conducted in accordance with the Declaration of Helsinki (as revised in 2013). Data were obtained after this study had been approved by the Ethical Review Committee of the Affiliated Cancer Hospital of Zhengzhou University (No. 2019001). As this study was retrospectively designed, informed consent was waived by the Affiliated Cancer Hospital of Zhengzhou University.

Open Access Statement: This is an Open Access article distributed in accordance with the Creative Commons Attribution-NonCommercial-NoDerivs 4.0 International License (CC BY-NC-ND 4.0), which permits the noncommercial replication and distribution of the article with the strict proviso that no changes or edits are made and the original work is properly cited (including links to both the formal publication through the relevant DOI and the license). See: https://creativecommons.org/licenses/by-nc-nd/4.0/.

\section{References}

1. Cortazar P, Zhang L, Untch M, et al. Pathological complete response and long-term clinical benefit in breast cancer: the CTNeoBC pooled analysis. Lancet 2014;384:164-72.

2. Curigliano G, Burstein HJ, Winer EP, et al. De-escalating and escalating treatments for early-stage breast cancer: the St. Gallen International Expert Consensus Conference on the Primary Therapy of Early Breast Cancer 2017. Ann Oncol 2017;28:1700-12.

3. Sanchez AM, Franceschini G, Scardina L, et al. Current weaknesses of breast conserving strategies after neoadjuvant chemotherapy in breast cancer treatment. Transl Cancer Res 2018;7:S356-64.

4. Kantor O, Sipsy LM, Yao K, et al. A Predictive Model for Axillary Node Pathologic Complete Response after Neoadjuvant Chemotherapy for Breast Cancer. Ann Surg Oncol 2018;25:1304-11.

5. van la Parra RF, Kuerer HM. Selective elimination of breast cancer surgery in exceptional responders: historical perspective and current trials. Breast Cancer Res 2016;18:28.

6. Hammond ME, Hayes DF, Dowsett M, et al. American Society of Clinical Oncology/College Of American Pathologists guideline recommendations for immunohistochemical testing of estrogen and progesterone receptors in breast cancer. J Clin Oncol 2010;28:2784-95.

7. Wolff AC, Hammond ME, Hicks DG, et al. Recommendations for human epidermal growth factor receptor 2 testing in breast cancer: American Society of Clinical Oncology/College of American Pathologists clinical practice guideline update. J Clin Oncol 2013;31:3997-4013.

8. Imyanitov EN, Yanus GA. Neoadjuvant therapy: theoretical, biological and medical consideration. Chin Clin Oncol 2018;7:55.

9. Mougalian SS, Hernandez M, Lei X, et al. TenYear Outcomes of Patients With Breast Cancer With Cytologically Confirmed Axillary Lymph Node Metastases and Pathologic Complete Response After Primary Systemic Chemotherapy. JAMA Oncol 2016;2:508-16.

10. Choi HJ, Ryu JM, Kim I, et al. Prediction of axillary pathologic response with breast pathologic complete response after neoadjuvant chemotherapy. Breast Cancer Res Treat 2019;176:591-6.

11. Gradishar WJ, Anderson BO, Balassanian R, et al. Breast Cancer, Version 4.2017, NCCN Clinical Practice Guidelines in Oncology. J Natl Compr Canc Netw 2018;16:310-20.

12. Tadros AB, Yang WT, Krishnamurthy S, et al. 
Identification of Patients With Documented Pathologic Complete Response in the Breast After Neoadjuvant Chemotherapy for Omission of Axillary Surgery. JAMA Surg 2017;152:665-70.

13. Barron AU, Hoskin TL, Day CN, et al. Association of Low Nodal Positivity Rate Among Patients With ERBB2-Positive or Triple-Negative Breast Cancer and Breast Pathologic Complete Response to Neoadjuvant Chemotherapy. JAMA Surg 2018;153:1120-6.

14. Samiei S, van Nijnatten TJA, de Munck L, et al. Correlation Between Pathologic Complete Response in the Breast and Absence of Axillary Lymph Node Metastases After Neoadjuvant Systemic Therapy. Ann Surg 2020;271:574-80.

15. Canavese G, Dozin B, Vecchio C, et al. Accuracy of sentinel lymph node biopsy after neo-adjuvant chemotherapy in patients with locally advanced breast cancer and clinically positive axillary nodes. Eur J Surg Oncol 2011;37:688-94.

16. Tee SR, Devane LA, Evoy D, et al. Meta-analysis of sentinel lymph node biopsy after neoadjuvant chemotherapy in patients with initial biopsy-proven nodepositive breast cancer. Br J Surg 2018;105:1541-52.

17. Shen J, Gilcrease MZ, Babiera GV, et al. Feasibility and accuracy of sentinel lymph node biopsy after preoperative chemotherapy in breast cancer patients with documented axillary metastases. Cancer 2007;109:1255-63.

18. Kuehn T, Bauerfeind I, Fehm T, et al. Sentinel-lymphnode biopsy in patients with breast cancer before and after neoadjuvant chemotherapy (SENTINA): a prospective, multicentre cohort study. Lancet Oncol 2013;14:609-18.

19. Boughey JC, Suman VJ, Mittendorf EA, et al. Sentinel lymph node surgery after neoadjuvant chemotherapy in patients with node-positive breast cancer: the ACOSOG

Cite this article as: Zhu J, Li J, Fan Z, Wang H, Zhang J, Yin Y, Fu P, Geng C, Jin F, Jiang Z, Liu Z. Association of higher axillary pathologic complete response rate with breast pathologic complete response after neoadjuvant chemotherapy. Ann Transl Med 2020;8(16):992. doi: 10.21037/atm-20-5172
Z1071 (Alliance) clinical trial. JAMA 2013;310:1455-61.

20. Boileau JF, Poirier B, Basik M, et al. Sentinel node biopsy after neoadjuvant chemotherapy in biopsy-proven nodepositive breast cancer: the SN FNAC study. J Clin Oncol 2015;33:258-64.

21. Caudle AS, Yang WT, Krishnamurthy S, et al. Improved Axillary Evaluation Following Neoadjuvant Therapy for Patients With Node-Positive Breast Cancer Using Selective Evaluation of Clipped Nodes: Implementation of Targeted Axillary Dissection. J Clin Oncol 2016;34:1072-8.

22. Murphy BL, Fazzio RT, Hoskin TL, et al. Management of the axilla in metaplastic breast carcinoma. Gland Surg 2018;7:200-6.

23. Schaefgen B, Mati M, Sinn HP, et al. Can Routine Imaging After Neoadjuvant Chemotherapy in Breast Cancer Predict Pathologic Complete Response? Ann Surg Oncol 2016;23:789-95.

24. Keune JD, Jeffe DB, Schootman M, et al. Accuracy of ultrasonography and mammography in predicting pathologic response after neoadjuvant chemotherapy for breast cancer. Am J Surg 2010;199:477-84.

25. Wang $\mathrm{Y}$, Zhang C, Liu J, et al. Is $18 \mathrm{~F}-\mathrm{FDG}$ PET accurate to predict neoadjuvant therapy response in breast cancer? A meta-analysis. Breast Cancer Res Treat 2012;131:357-69.

26. Kuerer HM, Rauch GM, Krishnamurthy S, et al. A Clinical Feasibility Trial for Identification of Exceptional Responders in Whom Breast Cancer Surgery Can Be Eliminated Following Neoadjuvant Systemic Therapy. Ann Surg 2018;267:946-51.

(English Language Editor: J. Reynolds) 


\section{Supplementary}

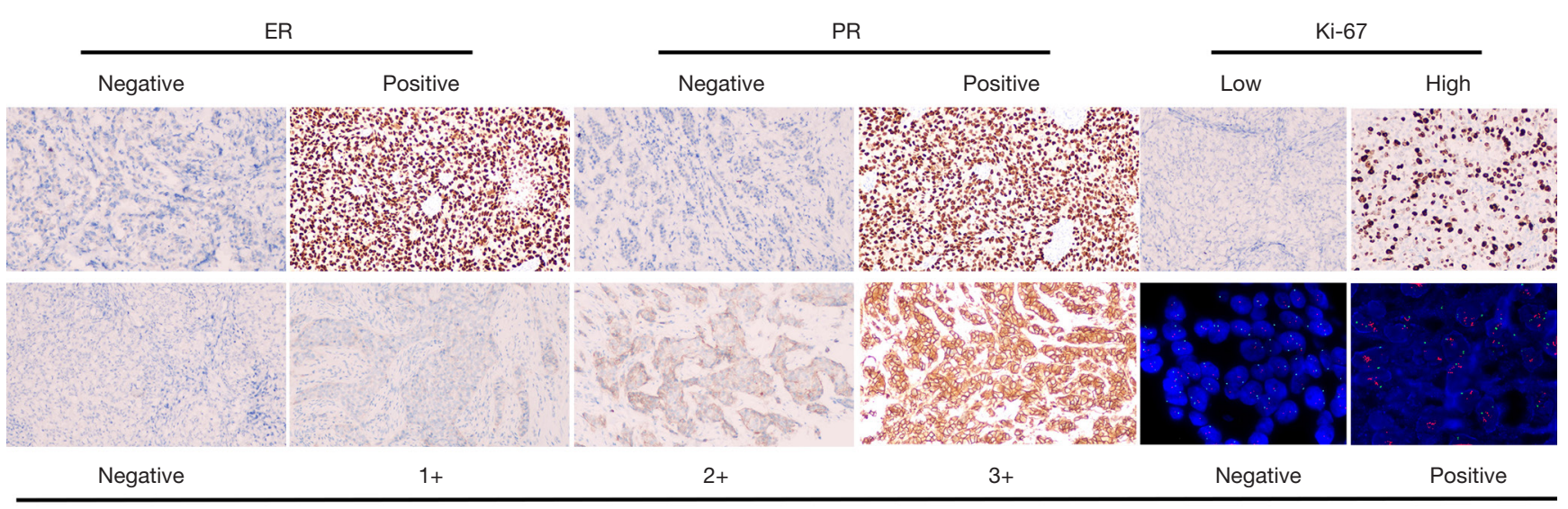

HER2

Figure S1 Immunohistochemical staining for ER, PR, HER2 Ki-67 and fluorescence in situ hybridization (FISH) for HER2 (100×). 